\title{
Background fitting of Fermi gamma-ray burst 091030613
}

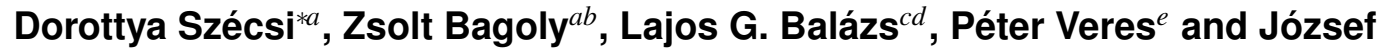 \\ Kóbori $^{a}$ \\ ${ }^{a}$ Dept. of Physics of Complex Systems, Eötvös University, H-1117 Budapest, Pázmány P. s. 1/A, \\ Hungary \\ ${ }^{b}$ Dept. of Physics, Bolyai Military University, H-1581 Budapest, POB 15, Hungary \\ ${ }^{c}$ Dept. of Astronomy, Eötvös University, H-1117 Budapest, Pázmány P. s. 1/A, Hungary \\ ${ }^{d}$ MTA CSFK Konkoly Observatory, H-1525 Budapest, POB 67, Hungary \\ ${ }^{e}$ Dept. of Astronomy \& Astrophysics, Pennsylvania State University, PA-16802 University Park, \\ 525 Davey Lab, USA \\ E-mail: szdpadteinf.elte.hu
}

Fermi Gamma-ray Burst Monitor (GBM) detects gamma-rays in the energy range $8 \mathrm{keV}-$ $40 \mathrm{MeV}$. Background fitting of the Fermi data is not trivial in some cases, especially when an Autonomous Repoint Request (ARR) is received. One good example is the burst 091030613 measured by the GBM, which cannot be fitted properly by a third-order polynomial of time. We present the background fitting of this burst for energy channels given in the CTIME data file. Our method is based on the motion of the satellite: we define three underlying parameters which depend on the actual position and orientation of the satellite and use them to fit the background. The main steps and results of this process are shown on the poster for the triggered NaI detector ' 3 '.

Gamma-Ray Bursts 2012 Conference-GRB2012,

May 07-11, 2012

Munich, Germany

\footnotetext{
* Speaker.
} 


\section{Summary of the method}

One only can perform a multitude of analyses of the Fermi data, if there is a satisfying background subtraction (for example, decision between long and short bursts require precise $\mathrm{T}_{90}$ measurments [1]). Since in the case of burst 091030613, fitting with a low-order polynomial function of time does not yield a satisfying result [3], we were looking for other variables, which can better describe the varying background.

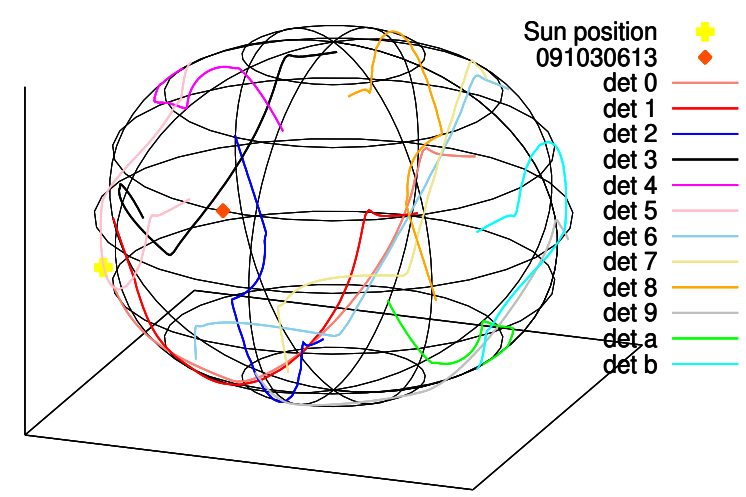

Figure 1: Directions of the $12 \mathrm{NaI}$ detectors' normal vector on the sky (in the second equatorial system), during the pre- and post- 1000 seconds around the burst 091030613. Sun's position: $\alpha_{S}=210.12^{\circ}, \delta_{S}=-13.00^{\circ}$ Burst position: $\alpha_{b}=260.72^{\circ}, \delta_{b}=22.67^{\circ}$

Using the LAT Spacecraft position files [5], we plotted the actual orientation of the $12 \mathrm{NaI}$ detectors during the burst, see Figure 1. One can see how the detectors are 'jumping' all over the sky: this is caused by the fast rotation of the satellite. Considering this behaviour, it is not surprising that the backgrounds of the lightcurves are very complicated to fit.

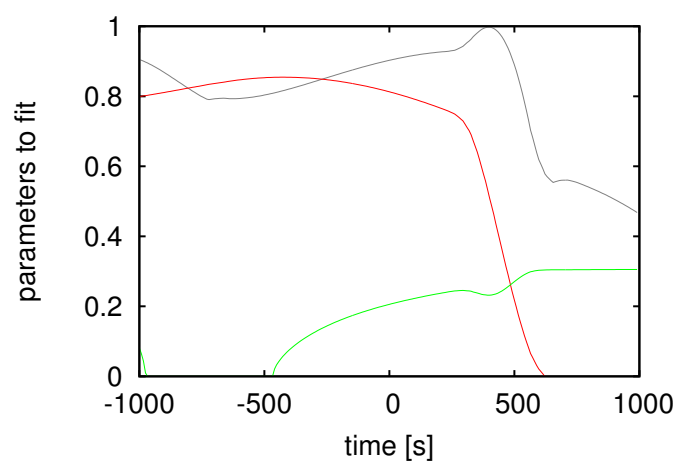

Figure 2: We use three underlying parameters: cosine of the Celestial Distance of the Burst's position and the Detector's normal vector (grey); cosine of the Celestial Distance of the Burst and the Sun (as most significant source of gamma background, red); the ratio of the Earth-uncovered Sky to the Detector Total Field of View (green). 


\section{Results}
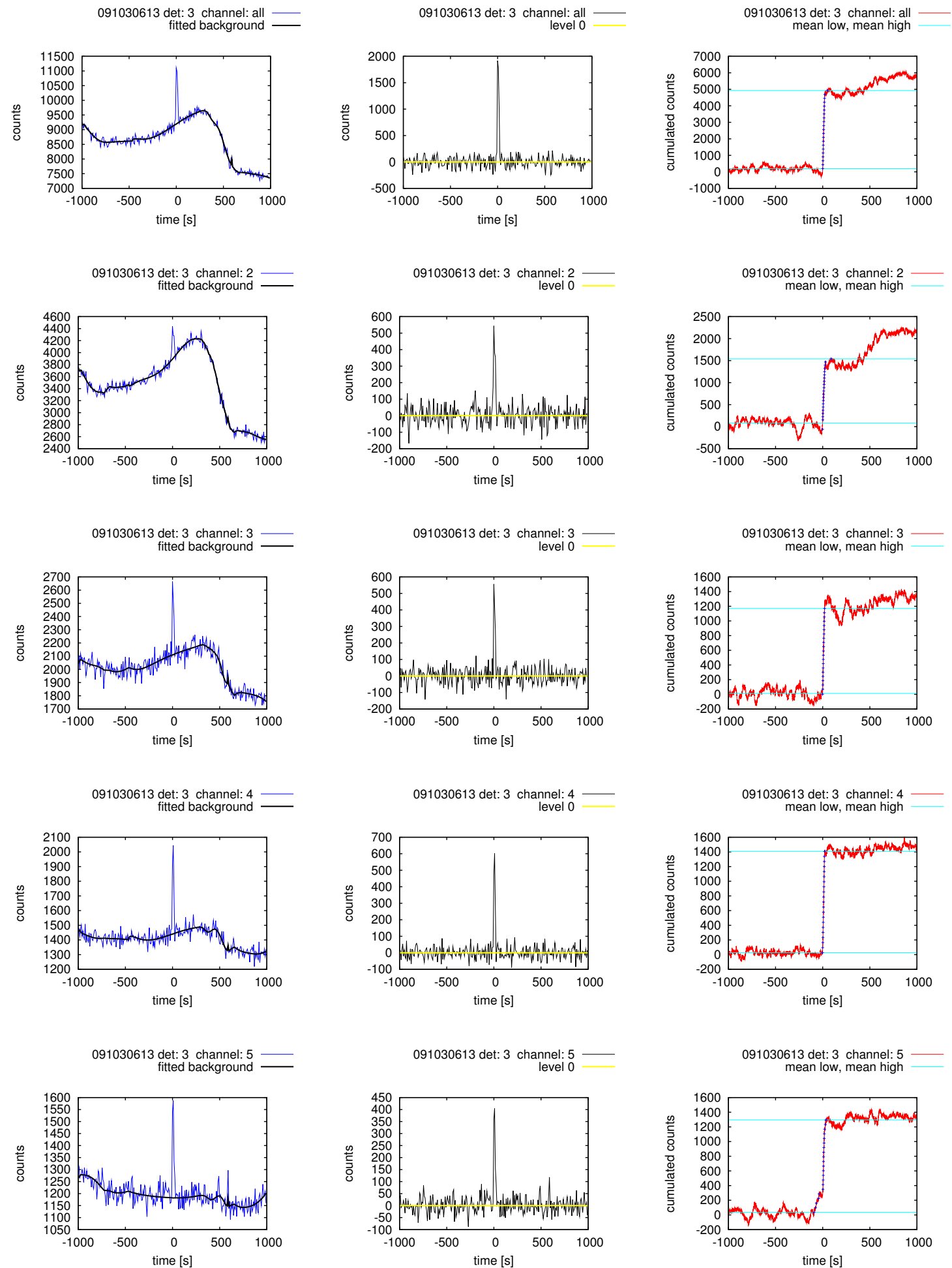

Figure 3: Result of the direction dependent background fitting. Rows from up to down: Sum of channels (11.50-982.23 keV); Channel 2 (11.50-26.63 keV); Channel 3 (26.63-50.43 keV); Channel 4 (50.43$102.38 \mathrm{keV})$; Channel 5 (102.39-295.74 keV). 
If one takes a look to the unfiltered lightcurves shown in blue lines in the left column on Figure 3, one can see the burst at $\mathrm{T}=0$ and the varying background. In order to fit and subtract this, we defined three underlying variables (see on Figure 2) based on the actual position and rotation of the Fermi. Using these, we fitted a 3rd order hypersurface (black line on left hand side on Figure 3) and subtracted from the data (lightcurves in the middle on Figure 3). The following formula was used:

$$
\begin{aligned}
& \operatorname{surface}(x, y, z)=A \cdot x^{3}+B \cdot x^{2}+C \cdot x+D \cdot y^{3}+E \cdot y^{2}+F \cdot y+G \cdot z^{3}+H \cdot z^{2}+I \cdot z+J \cdot x^{2} \cdot y+ \\
& +K \cdot x \cdot y^{2}+L \cdot x^{2} \cdot z+M \cdot x \cdot z^{2}+N \cdot y^{2} \cdot z+O \cdot y \cdot z^{2}+P \cdot x \cdot y \cdot z+Q \cdot x \cdot y+R \cdot x \cdot z+S \cdot y \cdot z+T,
\end{aligned}
$$

where $\mathrm{x}, \mathrm{y}$ and $\mathrm{z}$ are the three underlying variables described above, and the capital letters are fitted parameters. Fitting was done by the method of least squares, using Octave's pseudoinvert function [6].

At the right hand side on Figure 3, we plotted cumulated lightcurves and used them to measure duration of the burst ( $\mathrm{T}_{90}$ written at the bottom of the pictures). Fermi GBM Catalogue reports $\mathrm{T}_{90}^{\text {Cat. }}=19.2 \pm 0.9 \mathrm{~s}$ [4].

The GBM triggered detectors were '3' and ' 8 '. We analysed the lightcurves of detector ' 3 '. (On Figure 1 of the sky above, path of detector ' 3 ' is shown with black line.) CTIME files contain 8 channels between $4 \mathrm{keV}-2000 \mathrm{keV}$ but data of lowest and highest channels contain too much noise. Sum of channels 2-7, and channels 2, 3, 4, and 5 were plotted.

\section{Discussion}

First of all, we emphasise that we created a Fermi-specific method based on the motion of the satellite, which gave a good model for the background.

Sum of the channels give us a $\mathrm{T}_{90}^{\text {Sum }}=21.0 \mathrm{~s}$, which we can take as our final result for the duration of this burst. However, there is no physical evidence that a GRB has the same duration in every energy range, as the lightcurves of the different channels suggest $\left(\mathrm{T}_{90}^{3}=17.5 \mathrm{~s}\right.$ and $\left.\mathrm{T}_{90}^{4}=18.6 \mathrm{~s}\right)$. Furthermore, channels 2 and 5 show some strong deviation from the $\mathrm{T}_{90}^{\text {Sum }}$, probably because of a higher level of noise.

We note that since the fitting presented here has quite a complex algorithm, the measuring of errors is still under work and will be presented in a forthcoming article.

Channel 4 contains the highest rate of counts, and here our result $\left(\mathrm{T}_{90}^{4}=18.6\right)$ is consistent with the Fermi GBM Catalogue within the error limits.

\section{Acknowledgments}

This work was supported by the Hungarian OTKA-77795 grant.

\section{References}

[1] I. Horváth, A further study of the BATSE Gamma-Ray Burst duration distribution, A\&A 392 (2002) 791 [arXiv:astro-ph/0205004].

[2] C. Meegan et al., The Fermi Gamma-ray Burst Monitor, ApJ 702 (2009) 791 [arXiv: 0908 . 0450 ]. 
[3] D. Szécsi et al., GRB duration distribution considering the position of the Fermi, Acta Polytechnica 52 (2012) 43 [arXiv: 1202.2592 ].

[4] heasarc.gsfc.nasa.gov/W3Browse/fermi/fermigbrst.html

[5] fermi.gsfc.nasa.gov/cgi-bin/ssc/LAT/LATDataQuery.cgi

[6] www.gnu.org/software/octave/ 